\title{
BMJ Open The role of rehabilitation measures in reintegration of children with brain tumours or leukaemia and their families after completion of cancer treatment: a study protocol
}

\author{
Mona Leandra Peikert, Laura Inhestern, Corinna Bergelt
}

To cite: Peikert ML, Inhestern L, Bergelt C. The role of rehabilitation measures in reintegration of children with brain tumours or leukaemia and their families after completion of cancer treatment: a study protocol. BMJ Open 2017;7:e014505. doi:10.1136/ bmjopen-2016-014505

- Prepublication history for this paper is available online. To view these files please visit the journal online (http://dx.doi org/10.1136/bmjopen-2016014505).

Received 5 October 2016 Revised 24 May 2017 Accepted 25 May 2017

CrossMark

Department of Medical Psychology, University Medical Center Hamburg-Eppendorf, Hamburg, Germany

Correspondence to Mona Leandra Peikert; m.peikert@uke.de

\section{ABSTRACT}

Introduction For ill children as well as for their parents and siblings, childhood cancer poses a major challenge. Little is known about the reintegration into daily life of childhood cancer survivors and their families. The aim of this prospective observational study is to further the understanding of the role of rehabilitation measures in the reintegration process of childhood leukaemia or brain tumour survivors and their family members after the end of cancer treatment.

Methods and analysis This prospective observational study consists of three study arms: a quantitative study in cooperation with three German paediatric oncological study registries (study arm 1), a quantitative study in cooperation with a rehabilitation clinic that offers a familyoriented paediatric oncological rehabilitation programme (study arm 2) and a qualitative study at 12-month followup including families from the study arms 1 and 2 (study arm 3). In study arm 1, children, parents and siblings are surveyed after treatment (baseline), 4-6 months after baseline measurement and at 12-month follow-up. In study arm 2, data are collected at the beginning and at the end of the rehabilitation measure and at 12-month follow-up. Families are assessed with standardised questionnaires on quality of life, emotional and behavioural symptoms, depression, anxiety, fear of progression, coping and family functioning. Furthermore, self-developed items on rehabilitation aims and reintegration into daily life are used. Where applicable, users and non-users of rehabilitation measures will be compared regarding the outcome parameters. Longitudinal data will be analysed by means of multivariate analysis strategies. Reference values will be used for comparisons if applicable. Qualitative data will be analysed using thematic analysis. Ethics and dissemination This study has been approved by the medical ethics committee of the Medical Chamber of Hamburg. Data will be published in peer-reviewed journals and presented at conferences.

\section{INTRODUCTION}

Cancer diagnosis in children and adolescents leads to major disruption in their own lives and in the whole family. Depending
Strengths and limitations of this study

- The study includes users and non-users of a familyoriented rehabilitation programme and thus enables the investigation of facilitating factors and barriers for the utilisation of rehabilitation measures as well as its role in long-term reintegration processes.

- The inclusion of childhood cancer survivors, their parents and siblings allows for multiperspective analyses on long-term developments and factors influencing the families' reintegration into daily life after childhood cancer.

- The results of this study may help to identify specific needs for support after the end of cancer treatment and to optimise healthcare services that support the families with the re-entry into 'normal' life.

- Due to the mainly questionnaire-based study design and thus the required sufficient German language skills, a selection bias cannot be ruled out.

- Ethical guidelines and the effort to carry out a nationwide study require a complex recruitment scheme in which a subsequent non-responder analysis can only be conducted for medical data.

on diagnosis and treatment, children and adolescents are torn out of their daily life such as family and social life as well as school for months or even for years. Children and adolescents with cancer suffer from physical changes and display impaired quality of life and well-being compared with their healthy peers. ${ }^{1-3}$ Behavioural changes and difficulties in reintegrating in school can occur in consequence of cancer disease and treatment. ${ }^{4}$ Even after the end of cancer treatment, patients with childhood cancer show elevated emotional distress and have an increased risk for developing mental health problems. ${ }^{56}$

Likewise, parents and siblings of the patient are confronted with major changes and burden in their lives when a child is diagnosed with cancer. ${ }^{7}$ In addition to the 
life-threatening disease, separation of family members due to hospital stays, social isolation or financial difficulties impact families. ${ }^{9}$ Parents of patients with childhood cancer report post-traumatic stress and low quality of life. $^{1011}$ They experience fear of progression and are highly emotionally burdened. ${ }^{12}{ }^{13}$ Healthy siblings often experience the loss of parental attention and have to fulfil new responsibilities and roles within the family. ${ }^{14}$ They show emotional reactions such as elevated distress and anxiety levels as well as behavioural problems or difficulties in school. ${ }^{15}$

The most frequent cancer diagnoses in children under the age of 18 years in Germany are leukaemia and brain tumours. ${ }^{16}$ Due to improved treatment methods, the 5-year survival rate in Germany has increased to approximately $80 \%$ for patients with childhood cancer under the age of 15 years. ${ }^{16}$ However, patients with childhood cancer and their families are at risk for long-term consequences. In this study, the end of cancer treatment is defined as the end of intensive cancer treatments such as chemotherapy, radiation, surgery and stem cell transplantation or bone marrow transplantation. After the end of cancer treatment, patients and their families are discharged from structured treatment plans to a new daily life - a milestone for many families. They want to look forward and try to restore family life to normality as before the disease. ${ }^{17}$ While they are relieved surviving the disease, families describe returning to daily life as a difficult time. ${ }^{18}$ In particular, parents can experience feelings of exhaustion and inner emptiness after a long time of exceptional circumstances requiring them to function and to pull through. ${ }^{17}$ After a long period of absence from school for the children and from working life for the parents, re-entry and reintegration are the next steps after the end of cancer treatment. ${ }^{19}{ }^{20}$ Still, familial conflicts can impede scholastic reintegration. ${ }^{21}$

In the German rehabilitation system, two rehabilitation concepts are established to support patients with childhood cancer and their families after the inpatient cancer treatment. ${ }^{22}{ }^{23}$ Family-oriented rehabilitation was developed for patients with childhood cancer ( $\leq 15$ years), their parents and healthy siblings. ${ }^{22}$ The primary aim of the 4-week inpatient rehabilitation programme is to achieve the patients' rehabilitation goals and to ensure the treatment success. ${ }^{22}$ Therefore, multiprofessional therapeutic teams of physicians, clinical psychologists, social education workers and other professionals offer group or individual therapies and activities as required for all family members during the rehabilitation programme. ${ }^{22}$ For adolescent patients with cancer ( $>15$ years), a rehabilitation programme in small groups was developed to adapt to the specific developmental needs of adolescents (e.g. support with development of autonomy). ${ }^{22}$ In this rehabilitation programme, adolescents are not accompanied by their family members.

So far, only a few studies evaluated family-oriented rehabilitation programmes. Overall, a positive impact on quality of life and psychological symptoms in patients, siblings and parents could be found. ${ }^{324-28}$ In a recent study, a different evaluation approach has been pursued. The authors analysed 422 medical discharge summaries of children and adolescents who participated in a paediatric oncological rehabilitation measure. ${ }^{29}$ According to rehabilitation physicians' opinion, $86 \%$ of the children and adolescents achieved their rehabilitation goals such as the improvement of physical efficiency and peer group integration. However, until now there are no longitudinal studies with an adequate reference group, and previous studies focused primarily on aspects such as quality of life. ${ }^{2426} 28$ Thereby, parental fear of progression or family functioning have not been investigated in the context of family-oriented rehabilitation programmes. Furthermore, in Germany, there is no systematic data available on the participation rate in family-oriented paediatric cancer rehabilitation programmes. Expert ratings range from $30 \%$ to $100 \%$ (unpublished pilot data). Moreover, little is known about the reintegration of childhood cancer survivors and their families into daily life after the end of cancer treatment and the potential role of rehabilitation measures in the reintegration process. Therefore, the primary aim of this study is to further the understanding of the process of reintegration of paediatric cancer survivors and their family members after the end of cancer treatment and the role of rehabilitation measures in this process.

The main research questions are:

1. Reintegration: which factors impede or facilitate reintegration?

2. Use of rehabilitation measures: which factors impede or facilitate use of rehabilitation measures?

3. Rehabilitation process: which factors influence activity and participation of families during and after the rehabilitation measure?

4. Rehabilitation process: which treatments do patients and families receive during the rehabilitation measure and how are rehabilitation goals, treatments and goal attainment associated?

5. Effects of rehabilitation measures: which are the longterm effects of rehabilitation measures with regard to reintegration and psychosocial outcomes?

\section{METHODS AND ANALYSIS \\ Design}

In order to address the research questions with regard to the role of rehabilitation measures in the reintegration of children and their families after completion of cancer treatment mentioned above, we conduct a prospective observational study with a longitudinal design including a baseline measurement and two follow-up measurements. The study focuses on the most frequent childhood cancers in Germany ${ }^{16}$ and thus includes children with brain tumours or leukaemia and their families.

The investigation of factors associated with the use of rehabilitation measures and the role of rehabilitation measures for the reintegration after the end of cancer 
treatment requires the inclusion of a comparison group of non-users. Therefore, this study aims to include a consecutive sample of patients and families at the end of cancer treatment in order to include both subsequent users and non-users of rehabilitation measures.

The study will consist of three study arms:

- The first study arm is conducted as a quantitative study in cooperation with three nationwide German paediatric oncological study registries in which childhood cancer patients with high and low grade brain tumours as well as leukaemia are registered (main research focus: participation vs non-participation in rehabilitation measures).

- The second study arm is a quantitative study in cooperation with a rehabilitation clinic offering a family-oriented paediatric cancer rehabilitation programme (main research focus: rehabilitation process).

- The third study arm is a qualitative interview study including 20-25 families from study arms 1 and 2 (main research focus: deeper understanding of the reintegration processes).

\section{Measurement time points}

Study arm 1 (study registries)

- Baseline: end of cancer treatment

- Follow-up: 4-6 months after baseline survey

- 12-month follow-up: 12 months after first follow-up

Study arm 2 (rehabilitation clinic)

- Pre: beginning of the rehabilitation measure

- Post: end of the rehabilitation measure

- 12-month follow-up: 12 months after the end of the rehabilitation measure

Study arm 3 (qualitative study)

- Twelve-month follow-up

The different measurement time points in study arms 1 and 2 have been chosen for two reasons. First, the timing of the entry into a rehabilitation measure depends on the families and their specific situation. Consequently, we cannot survey families after the end of cancer treatment in study arm 2. Second, the follow-up measurement in study arm 1 is temporally delayed in comparison with the post measurement in study arm 2 because we want to give the families the chance to use a rehabilitation measure between the first two measurement time points in order to receive matching times of measurements for the last two measurements in both study arms.

Participants from study arm 1 who attend the rehabilitation measure in the cooperating rehabilitation clinic drop out of study arm 1 and subsequently are surveyed in study arm 2. An overview of the recruitment and procedure in the study arms 1 and 2 is displayed in figure 1 .

\section{Cooperation partners}

The recruitment of participants in study arm 1 is carried out in cooperation with the International HIT-MED Registry
(I-HIT-MED; University Medical Center Hamburg-Eppendorf; ClinicalTrials.gov Identifier: NCT02417324), the study registry of the SIOP-LGG 2004 study (SIOP-LGG; Medical Center Augsburg; ClinicalTrials.gov Identifier: NCT00276640) and the CoALL-study registry (CoALL; University Medical Center Hamburg-Eppendorf; ClinicalTrials.gov Identifier: NCT01228331). The treatment group in study arm 2 is recruited in the Rehabilitation Clinic Bad Oexen.

\section{Inclusion and exclusion criteria}

In study arms 1 and 2, children and adolescents $(\geq 11$ years) and their siblings ( $\geq 11$ years) as well as their parents are surveyed. Parents can be biological or social parents or other attachment figures. In study arm 1 , inclusion and exclusion criteria will be assessed by the study registries (age and diagnosis) and the healthcare providers in the clinics. The rehabilitation physicians will assess inclusion and exclusion criteria at the beginning of the rehabilitation measure in study arm 2.

\section{Inclusion criteria}

- Brain tumour or leukaemia diagnosis (patients with low grade brain tumours and their families are only included if the patient received radiation or chemotherapy)

- Patients' age under 18 years

- Signed informed consent

\section{Exclusion criteria}

- Different diagnosis than brain tumour or leukaemia

- Patients' age over 17 years

- Refusal of participation

- Physical and/or mental burden

- Cognitive limitations

- Insufficient language skills

\section{Recruitment and procedure}

Study arm 1

The study registries estimate the end of cancer treatment of their registered patients based on the information available (i.e. time of diagnosis, treatment protocol). They inform the clinic where the patient receives treatment about the study. Healthcare providers at the clinics inform the patients and their families about the study after the end of cancer treatment, ask them to participate and pass a data set containing an invitation and information letter as well as a consent form to contact the family. After patients or parents send the signed consent and their contact data to the Department of Medical Psychology (University Medical Center Hamburg-Eppendorf), the parents are sent a set of baseline questionnaires and consent forms for participation for all included family members. If the signed informed consent form to contact the families is not sent back within 4 weeks after contacting the clinic where the patient receives treatment, the study registries remind the healthcare providers in the clinics to contact or remind the families where necessary. 
Study arm 1

(study registries)

Study registries identify patients and inform the clinics
Study arm 2

(rehabilitation clinic)

\section{End of inpatient cancer treatment}

Healthcare providers at the clinics inform the families about the study and pass a consent form to contact the family

Informed consent to contact family

Department of Medical

Psychology sends questionnaires

and consent forms to families

Baseline: end of cancer treatment

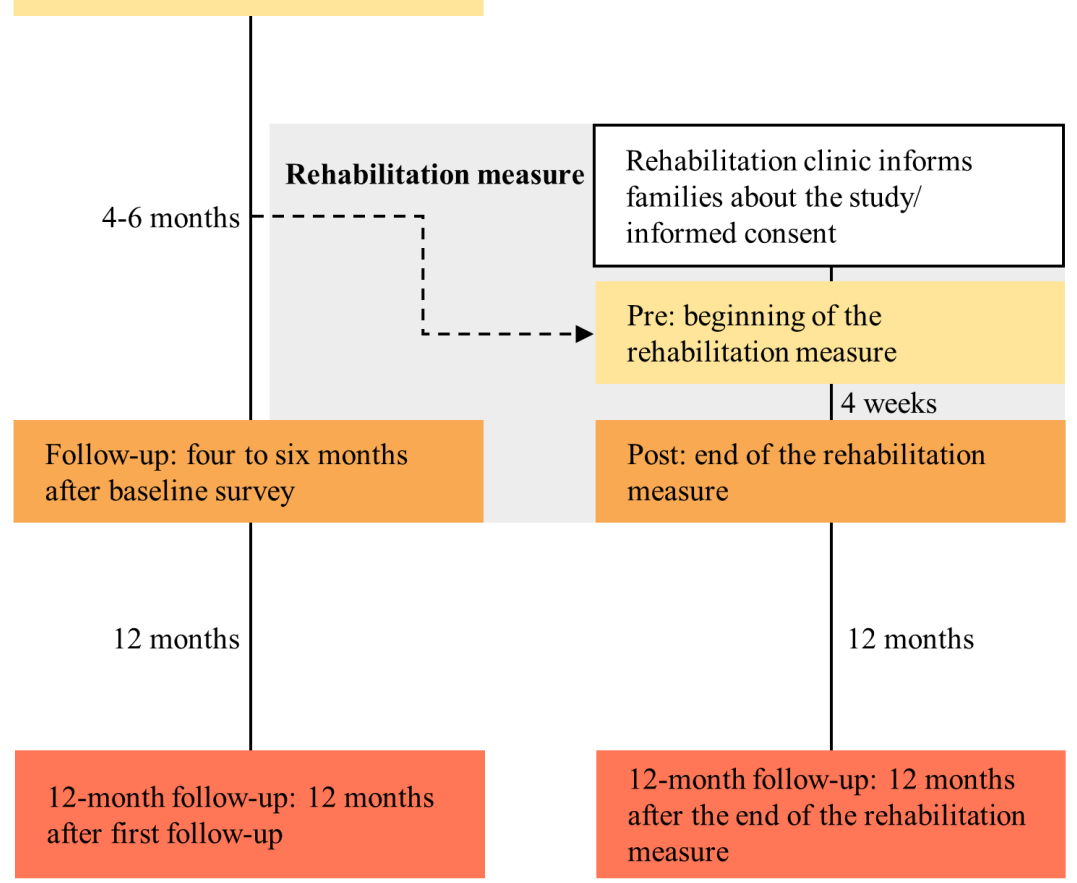

Figure 1. Study recruitment and procedure in study arms 1 and 2

Figure 1 Study recruitment and procedure in study arms 1 and 2 . 


\section{Study arm 2}

The rehabilitation clinic identifies patients with brain tumour or leukaemia at the beginning of the rehabilitation measure, informs them and their families about the study and asks them to participate. If the families agree to participate and sign the informed consent form, they receive a baseline questionnaire for all family members applicable according to the inclusion criteria. At the end of the rehabilitation measure, the families receive the second set of questionnaires. After 12 months, they receive the final set of questionnaires from the rehabilitation clinic.

If families agree to participate but have already been recruited into study arm 1 , the rehabilitation clinic informs the principal investigator and the family drops out of study arm 1.

\section{Study arm 3}

During the quantitative survey period patients from study arms 1 and 2 and their families are informed about the qualitative study. We conduct a consecutive sampling of the first 20-25 families that agree to participate in the interview study. We include multiple family members if both parents agree to participate. However, single parents or families with only one interested parent will also be included. If parents agree to be interviewed about 12-18 months after baseline measurement, they give their informed consent and contact data, where they can be reached via telephone. About 12-18 months after baseline measurement, one of the researchers is going to contact them and to conduct the telephone interview.

\section{Outcomes and measurements}

Study arms 1 and 2

\section{Quality of life \\ Children}

Children's health-related quality of life is assessed by the KINDL-R. ${ }^{30}$ There are three self-assessment versions (4-6 years, 7-13 years and 14-17 years) and two proxy versions for parents (3-6 years and 7-17 years). ${ }^{31}$ In this study, we use the self-assessment version for 14-17yearolds and the proxy version for parents of 7-17 year-olds. In an unpublished feasibility study, the version for 14-17 year-olds has also been used for children from 11 to 17 years of age without any difficulties. The KINDL-R includes 24 items covering six dimensions of quality of life (physical well-being, psychological well-being, self-esteem, family, friends and school) and six additional items on the dimension illness. ${ }^{32}$ Items refer to the past week and can be rated on a 5-point Likert scale from (1) never to (5) always. A total score and seven subscale scores can be calculated. The self-assessment version and the proxy version both have proved to be reliable and valid. ${ }^{33}$

\section{Parents}

The Ulm Quality of Life Inventory for Parents (ULQIE) is a 29-item self-report instrument, designed for measuring the quality of life of parents of chronically ill children on five subscales: functioning, satisfaction with family situation, emotional stress, self-fulfilment and general well-being. ${ }^{34}$ Parents assess on a 5-point Likert scale from (0) never to (4) always their quality of life over the past week. High values indicate a high quality of life. ${ }^{34} \mathrm{~A}$ total score of quality of life in general and five subscale scores can be obtained. The ULQIE has satisfactory psychometric properties. ${ }^{34}$

\section{Emotional and behavioural symptoms Children}

The Strengths and Difficulties Questionnaire is a 25-item instrument to assess strengths and difficulties in behaviour of children and adolescents on five different subscales: emotional symptoms, conduct problems, hyperactivity-inattention, peer relationship problems and prosocial behaviour. ${ }^{35}$ There are two versions: a self-report version for 11-16 year-olds and a version for parents and teachers of 4-16year-olds. ${ }^{35}$ Both versions use a 3-point Likert scale with (0) not true, (1) somewhat true and (2) certainly true. A total difficulties score as well as five subscale scores can be calculated. In this study, both versions are used to obtain a multiperspective view on the behaviour of patients with paediatric cancer and their siblings. The self-report version and the version for parents both have adequate psychometric properties. ${ }^{35} 36$

\section{Depression}

Parents

The 9-item depression module (PHQ-9) of the Patient Health Questionnaire (PHQ) is a self-report depression screening questionnaire. ${ }^{37}$ The PHQ-9 is used to classify depression severity in parents. The items reflect depression criteria based on the diagnosis criteria for depression disorders of the Diagnostic and Statistical Manual of Mental Disorders (DSM-IV). ${ }^{38}$ Answers can be given in four categories from (0) not at all to (3) nearly every day. ${ }^{39}$ The total score can be interpreted as the level of depression severity with cut-off values indicating minimal, mild, moderate, moderately severe and severe depression. ${ }^{39}$ The PHQ-9 has proved to be a valid and reliable questionnaire. ${ }^{3940}$

\section{Anxiety}

Parents

The Generalized Anxiety Disorder Screener (GAD-7) is a valid and reliable 7-item self-report instrument designed to identify probable cases of generalised anxiety disorder. ${ }^{414}$ In this study, the GAD-7 is used to classify symptoms of anxiety in parents. On a 4-point Likert scale from (0) not at all to (3) nearly every day subjects report how often they have been bothered by seven symptoms of the generalised anxiety disorder, derived from the DSM-IV diagnostic criteria A, B and C. ${ }^{38} 42$ By using cut-off values the subjects total GAD-7 score can be classified in mild, moderate and severe anxiety symptom levels. ${ }^{41}$ 


\section{Fear of progression}

\section{Parents}

To measure fear of progression in parents of patients with paediatric cancer, the adaption of the Fear of Progression Questionnaire for the parental perspective (FoP-Q-SF/ PR) is used in this study. ${ }^{43}$ The questionnaire contains nine items. Each of the items is scored on a 5-point Likert scale from (1) never to (5) very often. A total score can be calculated ${ }^{44}$ and a recommended cut-off value can be used for interpretation. ${ }^{43}$ The FoP-Q-SF/PR has adequate psychometric properties. $^{43}$

\section{Coping}

Children

Coping in patients with paediatric cancer and their siblings is measured by the KIDCOPE, ${ }^{45}$ which assesses 10 different coping strategies: distraction, social withdrawal, wishful thinking, self-criticism, blaming others, problem solving, emotional regulation, cognitive restructuring, social support and resignation. There are two versions of the KIDCOPE: a 15-item version for children (7-12.9 years) and a 11-item version for adolescents (13-18.9 years). ${ }^{45}$ In this study, we use the version for adolescents. Children rate the frequency of the coping strategy use on a 4-point Likert scale and the efficacy of each particular strategy on a 5-point Likert scale. ${ }^{45}$ The KIDCOPE has a sufficient reliability and validity. ${ }^{4546}$

\section{Parents}

Coping in parents is measured by the Coping Health Inventory for Parents (CHIP). ${ }^{47}$ The CHIP is used to assess the efficacy of different coping strategies in parents. $^{47}$ The 45 items are covering three different coping patterns: family (maintaining family integration, cooperation and an optimistic definition of the situation), support (maintaining social support, self-esteem and psychological stability) and medical (understanding the medical situation through communication with other parents and consultation with the medical staff). ${ }^{48}$ Ratings are made on a 4-point Likert scale from (0) not helpful to (3) extremely helpful. If parents did not use a certain coping strategy, they can specify if they did not use it or if they could not use it. ${ }^{47}$ A total score as well as subscale scores can be calculated. Higher scores indicate more helpful coping strategies. The reliability and validity of the CHIP have been proved. ${ }^{48}$

\section{Family functioning}

Parents and children assess the global family functioning on the general functioning subscale of the McMaster Family Assessment Device (FAD-GF). ${ }^{49}$ The subscale is composed of 12 items with a 4-point Likert scale from (1) strongly agree to (4) strongly disagree including aspects such as acceptance in the family and problem-solving behaviour. Higher total scores indicate lower family functioning. ${ }^{49}$ The FAD-GF has proved to be reliable and valid. $^{50}$

\section{Additional measures}

In addition to the validated scales listed above, we use self-developed items to assess parental burden related to the cancer illness, rehabilitation goals and their attainment, patient satisfaction or participant satisfaction respectively, reintegration and healthcare use. The self-developed items have been evaluated in an unpublished feasibility study. Received treatments during the rehabilitation measure and recommendations for aftercare are extracted from the medical discharge records in study arm 2. An overview of instruments, assessments and measurement time points (study arms 1 and 2) is displayed in table 1.

\section{Study arm 3}

The interviews will be conducted using an interview guideline developed on basis of theoretical background and knowledge from prior studies on similar topics. To test the comprehensibility of the questions, a pilot interview will be conducted. The interview guideline will cover the following aspects:

- How did the process of reintegration in school (for children) and working life (for parents) proceed?

- What are the experiences regarding facilitating factors and barriers for reintegration?

- If using rehabilitation measure: which factors were helpful for reintegration? Which aspects were amiss?

- Which healthcare services did parents and children use after completion of treatment?

\section{Data analyses}

\section{Quantitative analyses}

The research questions on reintegration, use of rehabilitation measures and the effects of rehabilitation measures (research questions 1, 2 and 5) will be analysed based on data from the combined sample from study arms 1 and 2. Where applicable, the sample will be divided into users and non-users of rehabilitation measures.

The research questions on the rehabilitation process (research questions 3 and 4) are analysed based on data from study arm 2 only.

Baseline scores and follow-up scores are analysed using descriptive analyses and if applicable compared with reference values. Using univariate analyses (t-test, analysis of variance) or comparable non-parametric tests, differences between subgroups (e.g. mothers and fathers, cancer patients and siblings, users and non-users of rehabilitation measures, patients with leukaemia and brain tumour) will be explored. Correlations will be calculated to explore associations between outcome variables and to measure potential non-independence of data in families.

The longitudinal nature of this study allows multivariate analysis strategies such as regression analysis, multilevel modelling and repeated measure analysis of variance to investigate the role of other factors and to examine changes over time. 
Table 1 Overview of study measures in study arms 1 and 2

\begin{tabular}{|c|c|c|c|c|}
\hline Measures & Instruments & Baseline/pre & 4-6 months/post & 12 months \\
\hline \multicolumn{5}{|l|}{$\begin{array}{l}\text { Data source: children } \\
\text { ( } \geq 11 \text { years) }\end{array}$} \\
\hline Quality of life & KINDL-R $\mathrm{R}^{30-33}$ & $\mathrm{X}$ & $\mathrm{X}$ & $\mathrm{X}$ \\
\hline $\begin{array}{l}\text { Emotional and behavioural } \\
\text { symptoms }\end{array}$ & $\begin{array}{l}\text { Strengths and Difficulties Questionnaire } \\
(\mathrm{SDQ})^{3536}\end{array}$ & $\mathrm{x}$ & $\mathrm{x}$ & $x$ \\
\hline Coping & KIDCOPE 4546 & $\mathrm{X}$ & $\mathrm{X}$ & $\mathrm{X}$ \\
\hline Family functioning & FAD-GF 4950 & $\mathrm{X}$ & $\mathrm{X}$ & $X$ \\
\hline Rehabilitation goals* & Self-developed items & $\mathrm{X}$ & & \\
\hline $\begin{array}{l}\text { Patient/participant } \\
\text { satisfaction* }\end{array}$ & Self-developed items & & $x$ & $x$ \\
\hline
\end{tabular}

\section{Data source: parent}

\begin{tabular}{|c|c|c|c|c|}
\hline Sociodemographic data & Self-developed items & $\mathrm{X}$ & & \\
\hline Medical data & $\begin{array}{l}\text { Self-developed items (e.g, parental } \\
\text { disease, parental healthcare use) }\end{array}$ & $\mathrm{X}$ & & \\
\hline Quality of life & $\begin{array}{l}\text { Ulm Quality of Life Inventory for } \\
\text { Parents } \\
\text { Pa }^{4}\end{array}$ & $\mathrm{x}$ & $\mathrm{X}$ & $x$ \\
\hline Depression & Patient Health Questionnaire 373940 & $x$ & $x$ & $\mathrm{X}$ \\
\hline Anxiety & $\begin{array}{l}\text { Generalised Anxiety Disorder } \\
\text { Screener }\end{array}$ & $\mathrm{x}$ & $\mathrm{X}$ & $x$ \\
\hline Fear of progression & FoP-Q-SF/PR ${ }^{43}$ & $\mathrm{x}$ & $\mathrm{X}$ & $x$ \\
\hline Coping & Coping Health Inventory for Parents ${ }^{4748}$ & $\mathrm{X}$ & $\mathrm{X}$ & $x$ \\
\hline Family functioning & FAD-GF 4950 & $\mathrm{X}$ & $\mathrm{X}$ & $\mathrm{x}$ \\
\hline $\begin{array}{l}\text { Parental burden related to the } \\
\text { cancer illness }\end{array}$ & Self-developed items & $\mathrm{X}$ & $x$ & $x$ \\
\hline Rehabilitation goals* & Self-developed items & $\mathrm{X}$ & & \\
\hline Participant satisfaction ${ }^{\star}$ & Self-developed items & & $X$ & $x$ \\
\hline Reintegration & Self-developed items & & $X$ & $x$ \\
\hline
\end{tabular}

About children (patient and max. two siblings)

\begin{tabular}{|c|c|c|c|c|}
\hline Sociodemographic data & Self-developed items & $X$ & & \\
\hline Medical data & $\begin{array}{l}\text { Self-developed items (e.g, diagnosis, } \\
\text { comorbidity, treatments received, use of } \\
\text { healthcare services) }\end{array}$ & $X$ & $\mathrm{X}$ & $x$ \\
\hline Quality of life & KINDL-R $R^{30-33}$ & $\mathrm{X}$ & $\mathrm{X}$ & $\mathrm{X}$ \\
\hline $\begin{array}{l}\text { Emotional and behavioural } \\
\text { symptoms }\end{array}$ & $\mathrm{SDQ}^{3536}$ & $X$ & $X$ & $\mathrm{X}$ \\
\hline $\begin{array}{l}\text { Reintegration and healthcare } \\
\text { use }\end{array}$ & Self-developed items & & $\mathrm{X}$ & $\mathrm{X}$ \\
\hline \multicolumn{5}{|l|}{ Medical assessment } \\
\hline $\begin{array}{l}\text { Medical data (diagnosis, } \\
\text { treatments and so on) } \dagger\end{array}$ & Self-developed items & $X$ & & \\
\hline Functional impairments $\ddagger$ & Self-developed items & $\mathrm{X}$ & $\mathrm{X}$ & \\
\hline Rehabilitation goals $\ddagger$ & Self-developed items & $\mathrm{X}$ & & \\
\hline Goal attainment $\ddagger$ & Self-developed items & & $\mathrm{X}$ & \\
\hline $\begin{array}{l}\text { Treatments received during } \\
\text { rehabilitation* }\end{array}$ & Rehabilitation discharge report & & $\mathrm{X}$ & \\
\hline $\begin{array}{l}\text { Recommendations for } \\
\text { aftercare * }\end{array}$ & Rehabilitation discharge report & & $\mathrm{X}$ & \\
\hline
\end{tabular}


Table 1 Continued

\begin{tabular}{|c|c|c|c|c|}
\hline Measures & Instruments & Baseline/pre & 4-6 months/post & 12 months \\
\hline
\end{tabular}

*Only in study arm 2.

†From study registries (study arm 1) or rehabilitation physician (study arm 2).

$\ddagger$ Assessed by rehabilitation physician (study arm 2).

FAD-GF, Family Assessment Device (General Functioning Scale; FoP-Q-SF/PR, Fear of Progression Questionnaire.

\section{Qualitative analyses}

Qualitative interviews (study arm 3) will be recorded and transcribed verbatim. Transcripts will be analysed using thematic analysis. ${ }^{5152}$ Main themes discussed in the interviews will be extracted in a first step. These themes will be subcategorised and discussed. A coding guideline with exemplary codes will be developed and presents the basis for a final coding of the entire material. To verify reliability and validity of the process and the final categories, two researchers will conduct parts of the coding independently and discuss their results.

\section{Sample size and power}

Sample size calculation indicates that a total sample size of 142 patients is sufficient to detect medium group differences $(\mathrm{f}=0.25)$ with a power of $95 \%$ at a significance level of $\mathrm{p}<0.05$. Considering two measurements, medium effects with a power of $85 \%$ can be detected with a sample size of 110 .

For study arm 1 (study registries), there is an expected patient number of about 224 per year (calculated based on previous annual statistics). Based on an estimated $50 \%$ response rate and a drop-out rate of $30 \%$ during follow-up, about 118 patients can be included within 18 months of recruitment. However, since the distribution of users and non-users of rehabilitation measures in study arm 1 is unknown and the study has an observational design with a focus on exploratory analyses, we aim to include as many patients as possible during the recruitment period.

For study arm 2 (rehabilitation measure), there is an expected patient number of about 117 per year (calculated based on previous annual statistics). Based on an estimated $50 \%$ initial response rate and a drop-out rate of $30 \%$ in 24 months of recruitment, about 82 patients can be included.

\section{ETHICS AND DISSEMINATION}

The medical ethics committee of the Medical Chamber of Hamburg reviewed and approved the study protocol (date: 20 May 2016, number: PV5277). Written information are provided to children ( $\geq 11$ years) and parents and they are asked to give their written informed consent prior to data collection. There are two different information and consent forms for children (11-13 years) and adolescents (14-17 years) with age-appropriate formulations. Parents additionally give their informed consent for their children ( $\geq 11$ years). The project is a naturalistic, observational study. There is no additional study-specific intervention besides the rehabilitation measure as a part of routine care within the framework of this study. The results of this project may help to optimise healthcare services that support the families after the end of cancer treatment with the re-entry into daily life.

The project duration is 42 months. The study was initiated in March 2016. Within the first 4 months, extensive preparatory work was carried out. The recruitment of participants in study arm 1 started in August 2016 and in study arm 2 in July 2016. Completion of data collection is planned to be in June 2019. Data entry, management and analysis as well as the publication of the findings in peer-reviewed journals and at conferences will take place continuously.

\section{DISCUSSION}

To the best of our knowledge, only few prospective, longitudinal studies have examined the situation of families with a child with cancer after the end of cancer treatment. ${ }^{19265354}$ So far, there is no study on processes of reintegration of all family members and no research on the role of rehabilitation measures in reintegration into daily life for affected families. The aim of this study is to investigate reintegration systematically and to explore factors associated with this process, especially focusing on rehabilitation measures. We aim to assess different trajectories of reintegration and to identify the role of rehabilitation measures for childhood cancer survivors and their families. The assessment of several outcomes from both the child's and the parent's view allows for a comprehensive, multiperspective insight in factors influencing reintegration. This might help to further the understanding of the situation of childhood cancer survivors and their families and to identify specific support needs with regard to reintegration. Therefore, the findings of this study may contribute to optimise healthcare services for families affected with childhood cancer and to develop aftercare programmes and interventions to facilitate the re-entry into 'normal' life of childhood cancer survivors and their families.

Acknowledgements This study is supported by the 'North Rhine-Westphalia Association for the Fight Against Cancer, Germany' (Arbeitsgemeinschaft für Krebsbekämpfung im Lande Nordrhein-Westfalen, ARGE). The authors acknowledge the teams from the cooperating study registries and the Rehabilitation Clinic Bad Oexen: (1) International HIT-MED Registry (I-HIT-MED; University Medical Center Hamburg-Eppendorf; contact person: Professor Dr Stefan Rutkowski), (2) SIOP-LGG 2004 study (SIOP-LGG; Medical Center Augsburg; contact person: Dr Astrid K. Gnekow), (3) CoALL-study registry (CoALL; University Medical Center HamburgEppendorf; contact persons: Professor Dr Martin Horstmann, PD Dr Gabriele 
Escherich), (4) Rehabilitation Clinic Bad Oexen (contact persons: Konstantin Krauth, Dr Thomas Schulte).

Contributors $\mathrm{CB}$ is the principal investigator of the study. $\mathrm{CB}$ and $\mathrm{LI}$ developed the study concept and the design. LI and MLP developed the study materials and acquire the data. MLP and LI will analyse and interpret the data. MLP and LI wrote the first draft of the study protocol. CB revised the first draft critically for important intellectual content. MLP, LI and CB have revised the subsequent drafts critically and approved the final manuscript to be published. MLP, LI and CB agree to be accountable for all aspects of the work. MLP and LI contributed equally to this article.

Funding The study is funded after peer review by the 'North Rhine-Westphalia Association for the Fight Against Cancer, Germany' (Arbeitsgemeinschaft für Krebsbekämpfung im Lande Nordrhein-Westfalen, ARGE). The ARGE is not involved in the study design, the collection, analysis and interpretation of data and in writing the manuscript.

Competing interests None declared.

Patient consent This article does not contain any personal medical information about an identifiable living individual.

Ethics approval Medical Ethics Committee of the Medical Chamber of Hamburg. Provenance and peer review Not commissioned; externally peer reviewed. Data sharing statement As this manuscript describes a study protocol, we cannot share any data yet.

Open Access This is an Open Access article distributed in accordance with the Creative Commons Attribution Non Commercial (CC BY-NC 4.0) license, which permits others to distribute, remix, adapt, build upon this work non-commercially, and license their derivative works on different terms, provided the original work is properly cited and the use is non-commercial. See: http://creativecommons.org/ licenses/by-nc/4.0/

(C) Article author(s) (or their employer(s) unless otherwise stated in the text of the article) 2017. All rights reserved. No commercial use is permitted unless otherwise expressly granted.

\section{REFERENCES}

1. Engelen V, Koopman HM, Detmar SB, et al. Health-related quality of life after completion of successful treatment for childhood cancer. Pediatr Blood Cancer 2011;56:646-53.

2. Reinfjell T, Lofstad GE, Nordahl HM, et al. Children in remission from acute lymphoblastic leukaemia: mental health, psychosocial adjustment and parental functioning. Eur J Cancer Care 2009;18:364-70.

3. Inhestern L, Beierlein V, Krauth KA, et al. Belastungen bei Eltern krebskranker Kinder in der familienorientierten Rehabilitation [Burden of parents of pediatric cancer patients in pediatriconcological rehabilitation]. Prax Kinderpsychol Kinderpsychiatr 2017:66:179-93.

4. Barrera M, Atenafu E. Cognitive, educational, psychosocial adjustment and quality of life of children who survive hematopoietic SCT and their siblings. Bone Marrow Transplant 2008;42:15-21.

5. Wenninger K, Helmes A, Bengel J, et al. Coping in long-term survivors of childhood cancer: relations to psychological distress. Psychooncology 2013;22:854-61.

6. Schrag NM, McKeown RE, Jackson KL, et al. Stress-related mental disorders in childhood cancer survivors. Pediatr Blood Cancer 2008;50:98-103.

7. Long KA, Marsland AL. Family adjustment to childhood cancer: a systematic review. Clin Child Fam Psychol Rev 2011; 14:57-88.

8. Pai AL, Greenley RN, Lewandowski A, et al. A meta-analytic review of the influence of pediatric cancer on parent and family functioning. $J$ Fam Psychol 2007;21:407-15.

9. McCaffrey CN. Major stressors and their effects on the well-being of children with cancer. J Pediatr Nurs 2006;21:59-66.

10. Kazak AE, Alderfer M, Rourke MT, et al. Posttraumatic Stress Disorder (PTSD) and Posttraumatic Stress Symptoms (PTSS) in families of adolescent childhood cancer survivors. $J$ Pediatr Psychol 2004;29:211-9.

11. Litzelman K, Catrine K, Gangnon R, et al. Quality of life among parents of children with cancer or brain tumors: the impact of child characteristics and parental psychosocial factors. Qual Life Res 2011;20:1261-9.
12. Kerr LM, Harrison MB, Medves J, et al. Understanding the supportive care needs of parents of children with cancer: an approach to local needs assessment. J Pediatr Oncol Nurs 2007;24:279-93.

13. Rodriguez EM, Dunn MJ, Zuckerman T, et al. Cancer-related sources of stress for children with cancer and their parents. J Pediatr Psychol 2012;37:185-97.

14. Alderfer MA, Long KA, Lown EA, et al. Psychosocial adjustment of siblings of children with cancer: a systematic review. Psychooncology 2010;19:789-805.

15. Houtzager BA, Grootenhuis MA, Last BF. Adjustment of siblings to childhood cancer: a literature review. Support Care in Cancer 1999;7:302-20.

16. Kaatsch P, Grabow D, Spix C. German Childhood Cancer Registry Annual Report 2016 (1980-2015): Institute of Medical Biostatistics, Epidemiology and Informatics (IMBEI) at the University Medical Center of the Johannes Gutenberg University Mainz. 2016.

17. McKenzie SE, Curle C. 'The end of treatment is not the end': parents' experiences of their child's transition from treatment for childhood cancer. Psychooncology 2012;21:647-54.

18. Labay LE, Mayans S, Harris MB. Integrating the child into home and community following the completion of cancer treatment. $J$ Pediatr Oncol Nurs 2004;21:165-9.

19. Hovén E, von Essen L, Norberg AL. A longitudinal assessment of work situation, sick leave, and household income of mothers and fathers of children with cancer in Sweden. Acta Oncol 2013;52:1076-85.

20. Wikman A, Hovén E, Cernvall M, et al. Parents of children diagnosed with cancer: work situation and sick leave, a five-year post end-oftreatment or a child's death follow-up study. Acta Oncol 2016;55(910):1152-7.

21. Ach E, Gerhardt CA, Barrera M, et al. Family factors associated with academic achievement deficits in pediatric brain tumor survivors. Psychooncology 2013;22:1731-7.

22. Arbeitsgemeinschaft Familienorientierte Rehabilitation (AGFOR). Positionspapier zur familienorientierten Rehabilitation bei krebskranken Kindern. 2001. https://www.kinderkrebsinfo.de/ fachinformationen/psapoh/rehabilitation/index_ger.html. (accessed Apr 2017).

23. Schröder HM, Lilienthal S, Schreiber-Gollwitzer BM, et al. Psychosoziale Versorgung in der Pädiatrischen Onkologie und Hämatologie, 2013. http://www.awmf.org/leitlinien/detail/ll/025-002. html. (accessed Apr 2017).

24. Besier T, Hölling H, Schlack R, et al. Impact of a family-oriented rehabilitation programme on behavioural and emotional problems in healthy siblings of chronically ill children. Child Care Health Dev 2010;36:686-95.

25. Häberle H, Schwarz R, Mathes L. Familienorientierte Betreuung bei krebskranken Kindern und Jugendlichen [Family orientated interventions of children and adolescents with cancer disease]. Prax Kinderpsychol Kinderpsychiatr 1997;46:405-19.

26. West CA, Besier T, Borth-Bruhns T, et al. Effectiveness of a familyoriented rehabilitation program on the quality of life of parents of chronically ill children. Klin Padiatr 2009;221:241-6.

27. van Buiren $\mathrm{M}$, Häberle $\mathrm{H}$, Mathes $\mathrm{L}$, et al. Rehabilitation von Familien krebskranker Kinder und Jugendlicher - Psychosozialer Langzeitverlauf [Rehabilitation of families of childhood cancer patients - long-term psychosocial follow-up]. Klin Padiatr 1998;210:304-9.

28. Goldbeck L, Hölling I, Schlack R, et al. The impact of an inpatient family-oriented rehabilitation program on parent-reported psychological symptoms of chronically ill children. Klin Padiatr 2011;223:79-84.

29. Inhestern L, Beierlein V, Krauth KA, et al. Belastungen und RehaZiele von Familien in der pädiatrisch-onkologischen Rehabilitation [Burden and rehabilitation goals of families in pediatriconcological rehabilitation]. Prax Kinderpsychol Kinderpsychiatr 2017;66:194-208

30. Ravens-Sieberer U, Bullinger M. Der Kindl-R Fragebogen zur Erfassung der gesundheitsbezogenen Lebensqualität bei Kindern und Jugendlichen - Revidierte Form. In: Schumacher J, Klaiberg A, Brähler E, eds. Diagnostische Verfahren zu Lebensqualität und Wohlbefinden. 1st ed. Göttingen: Hogrefe, 2003:184-8.

31. Ravens-Sieberer U, Bullinger M. KINDL-R. Questionnaire for measuring health-related quality of life in children and adolescents. Revised version. Manual. 2000. https://www.kindl.org/english/ manual/ (accessed March 2017).

32. Ellert U, Ravens-Sieberer U, Erhart M, et al. Determinants of agreement between self-reported and parent-assessed quality of life for children in Germany - results of the German Health Interview and Examination Survey for Children and Adolescents (KiGGS). Health Qual Life Outcomes 2011:9:102. 
33. Erhart M, Ellert U, Kurth BM, et al. Measuring adolescents' HRQoL via self reports and parent proxy reports: an evaluation of the psychometric properties of both versions of the KINDL-R instrument. Health Qual Life Outcomes 2009;7:77.

34. Goldbeck L, Storck M. Das Ulmer Lebensqualitäts-Inventar für Eltern chronisch kranker Kinder (ULQIE) [ULQIE: a quality-of-life inventory for parents of chronically ill children]. Z Klin Psychol Psychother 2002;31:31-39.

35. Klasen $\mathrm{H}$, Woerner W, Rothenberger A, et al. Die deutsche Fassung des Strengths and Difficulties Questionnaire (SDQ-Deu) - Übersicht und Bewertung erster Validierungs- und Normierungsbefunde [German version of the Strength and Difficulties Questionnaire (SDQGerman) - overview and evaluation of initial validation and normative results]. Prax Kinderpsychol Kinderpsychiatr 2003;52:491-502.

36. Woerner W, Becker A, Friedrich C, et al. Normierung und Evaluation der deutschen Elternversion des Strengths and Difficulties Questionnaire (SDQ): Ergebnisse einer repräsentativen Felderhebung [Normative data and evaluation of the German parent-rated Strengths and Difficulties Questionnaire (SDQ): results of a representative field study]. Z Kinder Jugendpsychiatr Psychother 2002;30:105-12.

37. Löwe B, Kroenke K, Herzog W, et al. Measuring depression outcome with a brief self-report instrument: sensitivity to change of the Patient Health Questionnaire (PHQ-9). J Affect Disord 2004;81:61-6.

38. American Psychiatric Association. Diagnostic and Statistical Manual of Mental Disorders: DSM-IV-TR. 4th ed. Washington, DC: American Psychiatric Association, 2000.

39. Kroenke K, Spitzer RL, Williams JB. The PHQ-9: validity of a brief depression severity measure. J Gen Intern Med 2001;16:606-13.

40. Martin A, Rief W, Klaiberg A, et al. Validity of the Brief Patient Health Questionnaire Mood Scale (PHQ-9) in the general population. Gen Hosp Psychiatry 2006;28:71-7.

41. Spitzer RL, Kroenke K, Williams JB, et al. A brief measure for assessing generalized anxiety disorder: the GAD-7. Arch Intern Med 2006;166:1092-7.

42. Löwe B, Decker $\mathrm{O}$, Müller $\mathrm{S}$, et al. Validation and standardization of the Generalized Anxiety Disorder Screener (GAD-7) in the general population. Med Care 2008;46:266-74.

43. Schepper F, Abel K, Herschbach P, et al. Progredienzangst bei Eltern krebskranker Kinder: Adaptation eines Fragebogens und Korrelate [Fear of progression in parents of children with cancer: adaptation of the fear of Progression Questionnaire and correlates]. Klin Padiatr 2015;227:151-6.

44. Mehnert A, Herschbach P, Berg P, et al. Progredienzangst bei Brustkrebspatientinnen - Validierung der Kurzform des Progredienzangstfragebogens PA-F-KF [Fear of progression in breast cancer patients - validation of the short form of the Fear of Progression Questionnaire (FoP-Q-SF)]. Z Psychosom Med Psychother 2006;52:274-88

45. Rathner G, Zangerle M. Copingstrategien bei Kindern und Jugendlichen mit Diabetes mellitus: Die deutschsprachige Version des KIDCOPE [Coping strategies of children and adolescents with diabetes mellitus: the german language version of KIDCOPE]. Z Klin Psychol Psychiatr Psychother 1996;44:49-74.

46. Spirito A, Stark LJ, Williams C. Development of a brief coping checklist for use with pediatric populations. J Pediatr Psychol 1988;13:555-74.

47. McCubbin HI, McCubbin MA, Cauble AE, et al. Fragebogen zur elterlichen Krankheitsbewältigung: Coping Health Inventory for Parents (CHIP) - Deutsche Version [Coping Health Inventory for Parents (CHIP) - German version]. Kindh Entwickl 2001;10:28-35

48. McCubbin HI, McCubbin MA, Patterson JM, et al. CHIP. Coping Health Inventory for Parents: an assessment of parental coping patterns in the care of the chronically ill child. J Marriage Fam 1983;45:359-70.

49. Epstein NB, Baldwin LM, Bishop DS. The McMaster Family Assessment Device. J Marital Fam Ther 1983;9:171-80.

50. Kabacoff RI, Miller IW, Bishop DS, et al. A psychometric study of the McMaster Family Assessment Device in psychiatric, medical, and nonclinical samples. Journal of Family Psychology 1990;3:431-9.

51. Mayring P. Qualitative Inhaltsanalyse: Grundlagen und Techniken Qualitative content analysis: basics and techniques. 10th ed. Weinheim Basel: Beltz Verlag, 2008.

52. Braun V, Clarke V. Using thematic analysis in psychology. Qual Res Psychol 2006;3:77-101.

53. Jörngården $A$, Mattsson $E$, von Essen L. Health-related quality of life, anxiety and depression among adolescents and young adults with cancer: a prospective longitudinal study. Eur J Cancer 2007;43:1952-8.

54. Sawyer M, Antoniou G, Toogood I, et al. Childhood Cancer: a 4-year prospective study of the psychological adjustment of children and parents. J Pediatr Hematol Oncol 2000;22:214-20. 